\title{
HER-2/neu Genotype of Breast Cancer May Change in Bone Metastasis
}

\author{
Tamás LŐRINCZ1 , József TÓTH ${ }^{2}$, Gayane BADALIAN, ${ }^{1}$ József TÍMÁR, ${ }^{3}$ Miklós SZENDRŐI ${ }^{1}$ \\ ${ }^{1}$ Department of Orthopedics, Semmelweis University, Budapest, Hungary Departments of ${ }^{2}$ Human and Experimental \\ Pathology and ${ }^{3}$ Tumor Progression, National Institute of Oncology, Budapest, Hungary
}

\begin{abstract}
The genotype of breast cancer (BRC) is considered to be relatively stable during tumor progression, accordingly, determination of the estrogen receptor and HER-2/neu status is currently based on the primary tumor. However, recent data suggest that the gene expression profile of the metastatic lesion can be different compared to that of the primary BRC. Accordingly, it is possible that the HER-2/neu status is different in the metastatic lesion and the primary BRC. Since the bone is the most frequent metastatic site during the progression of $B R C$, we have analyzed the HER-2/neu status of 48 bone metastatic BRC cases by immunohistochemistry and fluorescent in situ hybridization, and it was possible to compare it to the primary site in 23 cases. The fre-
\end{abstract}

Key words: breast cancer, bone metastatic, HER-2 quency of HER-2/neu amplification of BRC in the primary tumors was found to be $\mathbf{1 7 . 4 \%}$ compared to $10.5 \%$ in bone metastases. Half of BRC cases with HER-2/neu amplification lost this genotype in bone metastases (4/23 versus $2 / 23$, respectively) and even in the 2 cases where HER-2/neu amplification was retained in the metastases, the copy number was found to be decreased compared to the primary tumor. Based on our data and previous reports in the literature, we suggest to perform HER-2/neu testing both on primary tumor and samples obtained from BRC metastases, at least in case of primary tumors with HER-2/neu amplification, before introduction of HER-2/neu-targeting therapy. (Pathology Oncology Research Vol 12, No 3, 149-152)

\section{Introduction}

HER-2/neu oncoprotein is a transmembrane receptor, belonging to the epidermal growth factor receptor (EGFR) family, with tyrosine kinase activity. HER-2/neu has been shown to be overexpressed, most commonly by gene amplification, in a number of human malignancies, including breast cancer (BRC). ${ }^{1}$ Overexpression of the HER-2/neu oncoprotein in breast cancers is associated with shortened survival, enhanced aggressiveness, resistance to hormone- and chemotherapy ${ }^{2-4}$ and, eventually, decreased sensitivity to therapy. ${ }^{5}$ The extracellular domain of HER-2/neu is the target of trastuzumab (Herceptin $^{\circledR}$ ), the humanized anti-HER-2/neu monoclonal antibody. ${ }^{6}$ It has also been proved that the anticancer efficacy of trastuzumab is highly dependent on the HER-2/neu amplification of breast cancer. ${ }^{7}$ A careful selection of

Received: May 23, 2006; accepted: Aug 31, 2006

Correspondence: József TÍMÁR, MD, PhD, DSc, Department of Tumor Progression, National Institute of Oncology, Ráth György u. 7-9, Budapest, H-1122, Hungary. Tel.: 36-1-224-8786, Fax: 36-1224-8706, E-mail: jtimar@oncol.hu patients is crucial for increasing the clinical benefit of trastuzumab and avoiding unnecessary exposure of patients who most likely will not benefit from it. ${ }^{6}$ Immunohistochemistry (IHC) and fluorescence in situ hybridization (FISH) are standard methods to determine HER-2/neu protein expression and gene amplification, respectively. Clinical data indicate that patients with IHC $3+$ and/or FISH-positive BRC gain the greatest clinical benefit from Herceptin ${ }^{\circledR}$ treatment. ${ }^{8}$

It is still a matter of debate whether HER-2/neu status should be evaluated by early testing with systematically performed examinations on the primary tumor at the time of diagnosis of early breast cancer, or by pretreatment testing, i.e. performed on either the primary tumor or a metastatic site at the time of tumor relapse. ${ }^{9}$ To date, HER$2 /$ neu status in the majority of cases is evaluated in the primary tumor, since routine biopsy of metastatic sites is not a standard procedure. ${ }^{10}$ Nevertheless, the main targets of any systemic therapy in metastatic breast cancer are metastatic foci. ${ }^{10}$ Current diagnostics is based on the assumption that the genotype of primary BRC is similar to the one found in the metastases. Meanwhile, experimental and some clinical data suggest that 1) the primary tumor is 
genetically heterogeneous, 2) the clones responsible for organ dissemination may not even be present in the primary tumor but develop through sequential genetic alterations, and 3) they compose a very small percentage of the primary tumor. ${ }^{11}$ Earlier studies exclusively used HercepTest to assess the maintenance of HER-2/neu genotype in metastatic lesions and found it concordant in case of lymph node, liver and lung metastases. ${ }^{12-16}$ Recent reports provided evidence that the HER-2/neu genotype of BRC may change in the metastases: loss of HER-2/neu expression was reported in one case in pleural metastases, ${ }^{17}$ while loss of HER-2/neu amplification was found in $21 \%$, but appearance of HER-2/neu amplification was even more frequent in visceral metastases $(30 \%) .{ }^{18}$ Our aim was to analyze the HER-2/neu status of bone metastasis compared to the primary tumor in a larger cohort of BRC cases.

\section{Materials and Methods}

Patients

Bone metastatic samples of breast cancer were retrospectively collected from the pathology divisions of the following institutes: Departments of Orthopedics and Traumatology of Semmelweis University, National Institute of Traumatology. Samples derived from open biopsies of bone metastases obtained during transfocal stabilization of impending, complete pathological fractures, or resection of bone metastases. We excluded 9 cases because of overdecalcination or insufficient amount of tumor tissue in the sections. In total we investigated 48 metastatic cases. We were able to obtain paraffin-embedded samples of the primary breast cancers in 23 cases. The characteristics of the patients are shown in Table 1 . We have assessed the histological grade of the metastases in 44 out of the 48 investigated cases by the BloomRichardson method as previously described. ${ }^{19}$

Table 1. Characteristics of patients and tumors

\begin{tabular}{|c|c|}
\hline $\mathrm{n}$ & 48 \\
\hline Age (median) & 59 \\
\hline Sex (female/male) & $47 / 1$ \\
\hline Histology $(\mathrm{n}=48)$ & $\begin{array}{l}\text { invasive ductal carcinoma }(n=35) \\
\text { invasive lobular carcinoma }(n=8) \\
\text { other / not classified }(n=5)\end{array}$ \\
\hline $\begin{array}{l}\text { Grade of primary } \\
\text { tumors }(n=23)\end{array}$ & I: $1 / 23$ II: $10 / 23$ III: $12 / 23$ \\
\hline $\begin{array}{l}\text { Grade of bone } \\
\text { metastases }\left(n=44^{*}\right)\end{array}$ & I: $3 / 44$ II: 29/44 III: 12/44 \\
\hline
\end{tabular}

*In 4 out of 48 bone metastases the sample size was not sufficient for grading
Table 2. HER-2/neu status of breast cancers at the primary and metastatic site

\begin{tabular}{lcl}
\hline & IHC & FISH \\
\hline Primary tumors $\mathrm{n}=23$ & $3+: 2 / 23$ & A: $2 / 2$ \\
& $2+: 1 / 23$ & A: $1 / 1$ \\
& $0 / 1+: 20 / 23$ & A: $1 / 20$ \\
\hline Bone metastases $\mathrm{n}=48$ & $3+: 5 / 48$ & A: $4 / 5^{*}$ \\
& $2+: 4 / 48$ & A: $1 / 4$ \\
\hline
\end{tabular}

A: amplified. *: In one case we were not able to detect any signal, even with repeated analysis.

\section{Determination of the HER-2/neu status}

Immunohistochemistry (IHC). IHC was performed by using the HercepTest ${ }^{\mathrm{TM}}$ (DAKO, Glostrup, Denmark) according to the manufacturer's instruction, and as previously described. ${ }^{20}$ The slides were reviewed by two investigators. HER-2/neu staining was assessed semiquantitatively on a scale from 0 (negative) to $3+$ (strongly positive) according to standard protocols. ${ }^{20}$

Fluorescence in situ hybridization (FISH). FISH was performed in the cases where BRC had $2+$ or $3+$ HER$2 /$ neu IHC status in the bone metastases and/or in the primary tumors, or if discordance was found in HER-2/neu status detected by IHC between primary tumors and their corresponding bone metastases. FISH was performed by using the Oncor INFORM system (Ventana) as previously described, ${ }^{20}$ and by using the protocol No. 2 of the Benchmark automata stainer. Briefly, slides were deparaffinized and incubated with protease- 3 for 10 minutes. After denaturation at $90^{\circ} \mathrm{C}$ for 10 minutes, they were then incubated with one drop of INFORM HER-2/neu probe, followed by incubation with FITC/mouse anti-biotin and anti-mouse-Ig/FITC. In the next step slides were counterstained with 4,6-diamino-2-phenylindole (DAPI). Slides were assessed for HER-2/neu gene copy number as previously described: ${ }^{20}$ for each specimen, gene copy level was assessed in two areas of at least 20 non-overlapping tumor cell nuclei by using an epifluorescence microscope (Nikon Eclipse-600). A tumor was considered to contain gene amplification if there were more than four copies of HER-2/neu per cell. ${ }^{20}$

\section{Results}

Of the 48 metastatic lesions $9(18.75 \%)$ showed HER2/neu overexpression by IHC (3+: $5,2+: 4)$. We found HER-2/neu gene amplification in 1 out of the 4 IHC $2+$ cases, and in 4 out of the 5 IHC $3+$ cases (Table 2, Figure $1 c, d)$. In one of the IHC $3+$ cases we were not able to confirm the gene amplification even after repeated analysis. 

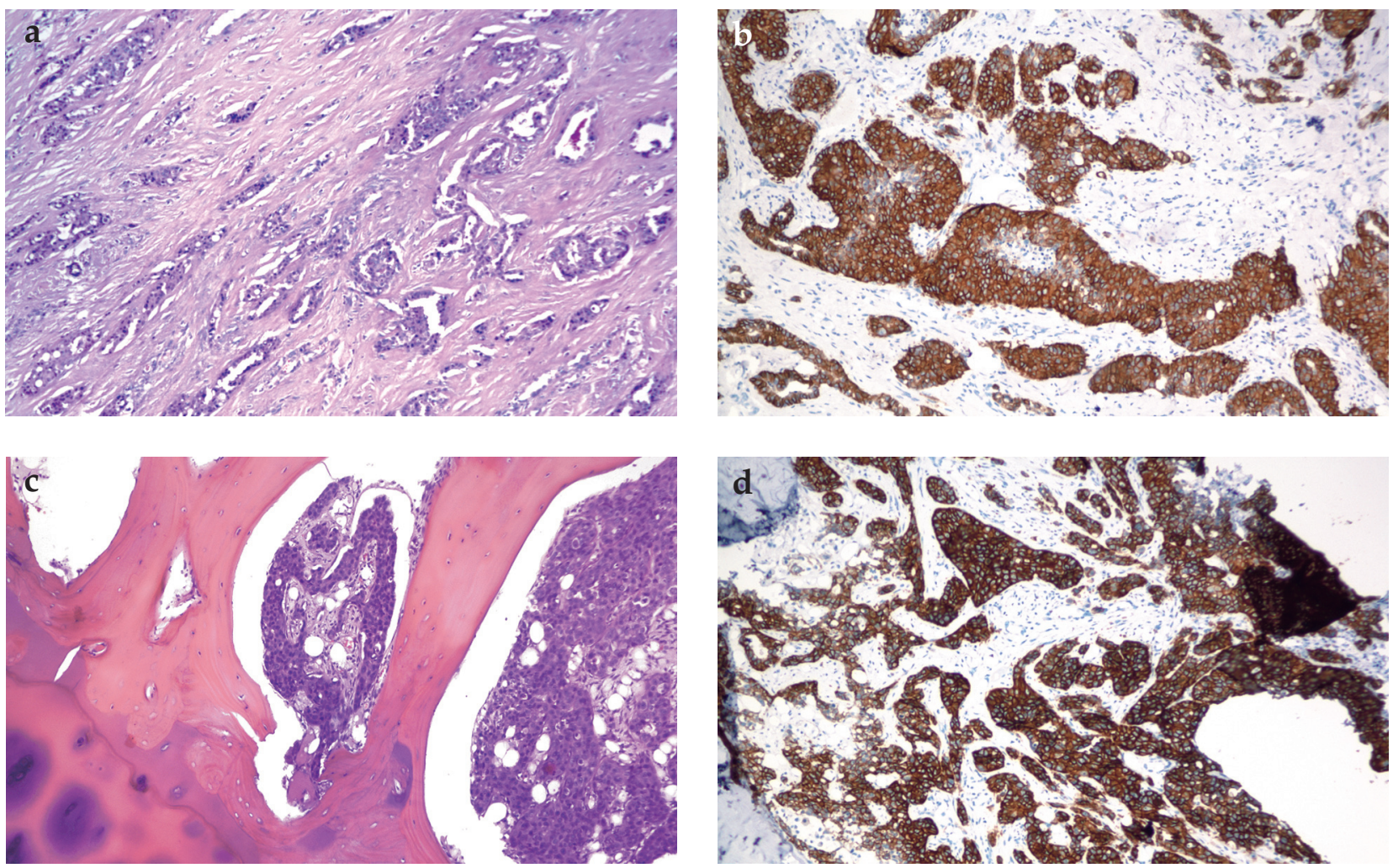

Figure 1. Overexpression of HER-2/neu protein in primary and bone metastatic BRC (case No 1). HER-2/neu protein was determined by immunohistochemistry using HercepTest. $\boldsymbol{a}$, $\boldsymbol{b}$ : primary tumor, c,d: bone metastasis. a, $\boldsymbol{c}$ : HEE-stained sections, $\boldsymbol{b}, \boldsymbol{d}$ : HER2/neu protein expression (3+). The overexpressed phenotype is maintained in the metastasis.

Taken together, five of the 48 bone metastatic breast cancer patients (10.5\%) had HER-2/neu gene amplification.

Twenty-three paired cases of primary and metastatic BRC tissue samples were available for comparative analysis. Using IHC and FISH we found that 4 out of the 23 primary tumors $(17.4 \%)$ had amplified HER-2/neu gene (Table 2, Figure $1 a, b)$. In bone metastases of the $23 \mathrm{BRC}$ we found only two cases where the HER-2/neu status was maintained while in two other cases the initial amplified status was changed to a

Table 3. Comparison of HER-2/neu status of BRC in bone metastases compared to the primary tumor

\begin{tabular}{lcclcccc}
\hline & \multicolumn{2}{c}{ Primary tumor } & & \multicolumn{2}{c}{ Bone metastasis } \\
\cline { 5 - 6 } Case no. & IHC & FISH & Status & IHC & FISH & Status \\
\hline 1 & $3+$ & $\mathrm{A}$ & + & $3+$ & $\mathrm{A}$ & + \\
2 & $3+$ & $\mathrm{A}$ & + & 0 & $\mathrm{~N}$ & - \\
3 & 0 & $\mathrm{~A}$ & + & $3+$ & $\mathrm{A}$ & + \\
4 & $2+$ & $\mathrm{A}$ & + & 0 & $\mathrm{~N}$ & - \\
\hline $5-23$ & $0 / 1+$ & n.t. & - & $0 / 1+$ & n.t. & - \\
\hline
\end{tabular}

$\mathrm{A}=$ amplified, $\mathrm{IHC}=$ immunohistochemistry, FISH $=$ fluorescence in situ hybridization, $\mathrm{N}=$ non-amplified, n.t. = not tested
HER-2/neu-negative one (Table 3). Furthermore, we also detected a lower HER-2/neu gene copy number in the bone metastases of the HER-2/neu amplified BRC compared to the primary tumor (Figure 2). On the other hand, we did not find alteration in the HER-2/neu status of the bone metastases of $\mathrm{BRC}$ where the primary tumor was normal (Table 3).

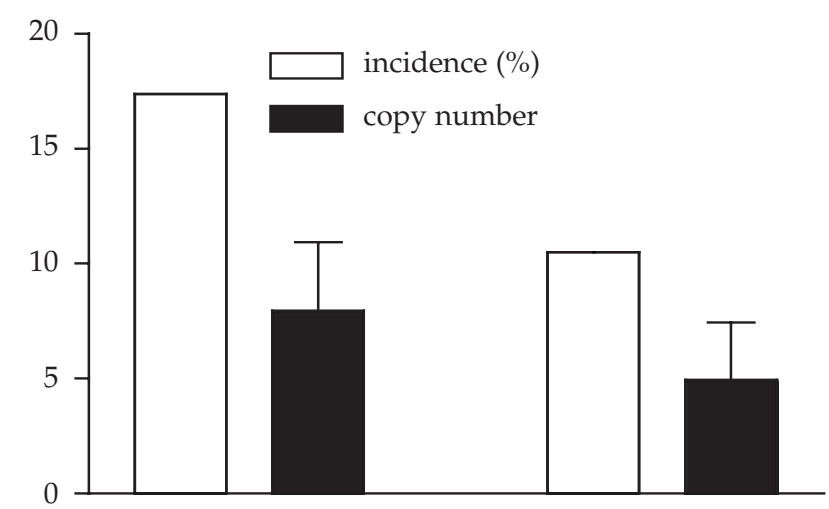

Figure 2. Comparison of HER-2/neu gene amplification in bone metastases and in the primary tumor of BRC, determined by FISH. Amplification incidence is expressed as \% of cases ( $n=23$ and $n=48$ for primary tumors and metastases, respectively), while copy number data are mean $\pm S E M$ ( $n=4$ and $n=5$ respectively). 


\section{Discussion}

There are reports that the estrogen receptor (ER) status of $\mathrm{BRC}$ is different in the primary tumor and bone metastases: usually the initial ER-positive phenotype reverts to an ER-negative one. ${ }^{21}$ A careful genetic analysis revealed a different cytogenetic profile of bone metastasis in BRC compared to the primary tumor. Furthermore, expression profiling also detected a unique gene signature in the bone metastases compared to other metastatic sites. ${ }^{22}$

Bone is one of the most frequently involved sites during breast cancer progression. ${ }^{23}$ It is, therefore, surprising that, with the exception of one study, ${ }^{10}$ the HER-2/neu genotype of bone metastasis was analyzed only in a very small number of patients $(<10) .{ }^{18,24,25}$ These case reports documented a relatively frequent change in HER-2/neu genotype in distant metastases in up to $20 \%$ of the cases. These data suggested that the common dogma that BRC maintains the geno/phenotype in metastasis may not be valid. Our recent findings further support these notions, revealing that in a small proportion of cases (about 10\%) the initial HER$2 /$ neu genotype may change. This may not seem a significant proportion of BRC cases, but it must be considered that in our cohort studies $50 \%$ of cases with HER-2/neu amplification lost their genotype in bone metastases. Unfortunately we were not able to perform a larger study on bone metastases because in approximately half of our cases we were not able collect the primary samples for comparison. We suggest that in the subset of BRC cases with HER-2/neu amplification the conversion of the genotype may occur more frequently compared to cases without amplification, which maintained their genotype in our study. Previous analyses on the HER-2/neu status of BRC at various metastatic sites indicated that the original genotype may change, either due to the selection process during tumor progression or under the pressure of anti-HER-2/neu treatment. ${ }^{12,16-18}$ However, the switch in genotype does not necessarily occur at all metastatic sites. ${ }^{17,18}$ Since the therapy of the HER-2/neu-amplified and non-amplified metastatic BRC is different, based on our data and on the literature we suggest to perform HER-2/neu testing both on primary tumor and metastases, at least in the case of primary tumors with HER-2/neu amplification. When HER2/neu status in metastatic BRC is negative at all locations tested, in other words, when there is no proof of maintained HER-2/neu amplification at any metastatic sites, there is no reason to apply anti-HER-2/neu therapy.

\section{References}

1. Mendelsohn J, Baselga J: The EGF receptor family as targets for cancer therapy. Oncogene 19: 6550-6565, 2000

2. Slamon DJ, Clark GM, Wong $S G$, et al: Human breast cancer: correlation of relapse and survival with amplification of the HER-2/neu oncogene. Science 235:177-182, 1987
3. Yu D, Hung MC: Overexpression of ErbB2 in cancer and ErbB2-targeting strategies. Oncogene 19: 6115-6121, 2000

4. Tsuda H, Hirohashi S, Shimotaso Y, et al: Correlation between long term survival in breast cancer patients and amplification of two putative oncogene co-amplification units: hst-1/int-2 and c-erbB-2/ear-1. Cancer Res 49:3104-3108, 1989

5. de Bono JS, Rowinsky EK: The ErbB receptor family: a therapeutic target for cancer. Trends Mol Med 8 (4 suppl): S19-S26, 2002

6. Ross JS, Fletcher JA: HER-2/neu (c-erb-B2) gene and protein in breast cancer. Am J Clin Pathol 112: 53-67, 1999

7. Cobleigh MA, Vogel CL, Triphaty D et al: Multinational study of the efficacy and safety of humanized anti-HER-2/NEU monoclonal antibody in woman who have HER-2/NEU-overexpressing metastatic breast cancer that has progressed after chemotherapy for metastatic disease. J Clin Oncol 17: 2639-2648, 1999

8. Smith IE: Efficacy and safety of Herceptin ${ }^{\circledR}$ in women with metastatic breast cancer: Result from pivotal clinical studies. Anticancer Drugs 12 (suppl 4): S3-S10, 2001

9. Di Leo A, Dowsett M, Horten B, et al: Current status of HER-2/neu testing. Oncology 63 (suppl 1): 25-32, 2002

10. Gancberg M,Di Leo A, Rouas $G$ et al: Comparison of topoisomerase IIa (topoII $\alpha$ ) and HER-2 status between primary $(\mathrm{P})$ breast cancer $(\mathrm{BC})$ and corresponding metastatic (M) sites. Ann Oncol 13: 1036-1043, 2002

11. Fidler IJ: Review: biologic heterogeneity of cancer metastasis. Breast Cancer Res Treat 9:17-26,1987

12. Masood S, Bui MM: Assessment of Her-2/neu overexpression in primary breast cancers and their metastatic lesions: an immunohistochemical study. Ann Clin Lab Sci 30: 259-265, 2000

13. Shimizu C, Fukutomi T, Tsuda H et al: c-erbB-2 protein overexpression and p53 immunoreaction in primary and recurrent breast cancer tissues. J Surg Oncol 73: 17-20, 2000

14. Carlsson J, Nordgren H, Sjöström J et al: HER-2/NEU expression in breast cancer primary tumours and corresponding metastases. Original data and literature review. Br J Cancer 90: 2344-2348, 2004

15. Vincent-Salomon A, Jouve $M$, Genin $P$ et al: HER-2/NEU status in patients with breast carcinoma is not modified selectively by preoperative chemotherapy and is stable during the metastatic process. Cancer 94: 2169-2173, 2002

16. Tanner M, Järvinen P, Isola J: Amplification of Her-2/neu and topoisomerase II $\alpha$ in primary and metastatic breast cancer. Cancer Res 61:5345-5348, 2001

17. Kunitomo K, Inoue S, Ichihara F et al: A case of metastatic breast cancer with outgrowth of HER-2/NEU-negative cells after eradication of HER-2/NEU-positive cells by humanized anti-HER-2/NEU monoclonal antibody (trastuzumab) combined with docetaxel. Hum Pathol 35: 379-381, 2004

18. Zidan J, Dashkovsky I, Stayerman $C$ et al: Comparison of HER-2 overexpression in primary breast cancer and metastatic sites and its effect on biological targeting therapy of metastatic disease. Br J Cancer 93: 552-556, 2005

19. Latinovic L, Heinze G, Birner P et al: Prognostic relevance of three histological grading methods in breast cancer. Int J Oncol 19: 12711277, 2001

20. Press MF, Slamon DJ, Flom KJ et al: Evaluation of HER-2/neu gene amplification and overexpression: comparison of frequently used assay methods in a molecularly characterized cohort of breast cancer specimens. J Clin Oncol 20: 3095-3105, 2002

21. Bruun Rasmussen B, Kamby C: Immunohistochemical detection of oestrogen receptors in paraffin sections from primary and metastatic breast cancer. Pathol Res Pract 185:856-859, 1989

22. Woelfle $U$, Cloos $J$ et al: Molecular signature associated with bone marrow micrometastasis in human breast cancer. Cancer Res 63:5679-5684, 2003

23. Yoneda T: Cellular and molecular mechanism of breast and prostate cancer metastasis to bone. Eur J Cancer 34: 240-245, 1998

24. Gong Y, Booser DJ, Sneige N: Comparison of HER-2 status determined by fluorescence in situ hybridization in primary and metastatic breast carcinoma. Cancer 103: 1763-1769, 2005

25. Regitnig $P$, Schippinger W, Lindbauer $M$ et al. Change of HER-2/neu status in a subset of distant metastases from breast carcinomas. J Pathol 203: 918-926, 2004 